\title{
AO Velorum: a young quadruple system with a ZAMS eclipsing BpSi primary ${ }^{\star}$
}

\author{
J. F. González ${ }^{1}$, S. Hubrig ${ }^{2}$, N. Nesvacil ${ }^{2,3}$, and P. North ${ }^{4}$ \\ ${ }^{1}$ Complejo Astronómico El Leoncito, Casilla 467, 5400 San Juan, Argentina \\ 2 European Southern Observatory, Casilla 19001, Santiago 19, Chile \\ 3 Department of Astronomy, University of Vienna, Tuerkenschanzstrasse 17, 1180 Vienna, Austria \\ ${ }^{4}$ Ecole Polytechnique Fédérale de Lausanne (EPFL), Laboratoire d'Astrophysique, Observatoire, 1290 Sauverny, Switzerland
}

Received 25 August 2005 / Accepted 11 November 2005

\section{ABSTRACT}

Using recent spectroscopic observations, we show that the triple system AO Vel with an eclipsing BpSi primary is in fact a remarkable quadruple system formed by two double-lined spectroscopic binaries with components close to the ZAMS. All available data have been reanalyzed to derive proper orbital parameters for both binary systems and to calculate absolute parameters of the eclipsing system. For the first time, direct determination of the radius and the mass have been obtained for a BpSi star. The study of the physical parameters of this unique system is especially important since it can be used as a test of evolutionary models for very young stars of intermediate mass.

Key words. stars: binaries: spectroscopic - stars: chemically peculiar - stars: fundamental parameters - stars: magnetic fields

\section{Introduction}

Upper main sequence stars with chemical peculiarities and large-scale organized magnetic fields constitute about $10 \%$ of A and B-type stars. To understand the physics of such stars, it is most important to identify the origin of their magnetic field. Two main hypotheses have been proposed: either these stars have retained the primordial field present at their formation, the ohmic decay being longer than the main sequence stellar lifetime (fossil field theory), or the field is generated and maintained by a contemporary dynamo. The first hypothesis implies that strongly magnetic stars must be found on the ZAMS (as well as over the whole width of the main sequence), while this is not necessarily true according to the second hypothesis. Hubrig et al. (2000) suggested that magnetic stars tend to accumulate in the middle of the main sequence strip if their mass is below $3 M_{\odot}$ (which would challenge the fossil field theory), while more massive magnetic stars seem more evenly distributed over the whole width of the main sequence, including near the ZAMS (Hubrig et al. 2005). However, the chemical peculiarities of these stars distort their colour indices, so that their effective temperatures suffer from an uncertainty which also propagates to other stellar parameters like e.g. the surface gravity (North 1998). Therefore, it is most important to

* Based on observations obtained at the European Southern Observatory, La Silla, Chile (ESO programmes Nos. 072.D-0235, 65.L-0316), and at the Complejo Astronómico El Leoncito (CASLEO), Argentina. determine the stellar parameters of magnetic stars in the most fundamental way, especially as the extent to which their evolution within the main sequence is identical to that of their nonmagnetic siblings is not known.

The most suitable systems for the accurate assessment of fundamental stellar parameters are double-lined eclipsing binaries. While a few double-lined spectroscopic binary systems (SB2) containing an Ap star of mass below $3 M_{\odot}$ are currently known, the rate of close binaries is much smaller among magnetic Bp stars (Gerbaldi et al. 1985; Carrier et al. 2002; North \& Debernardi 2004) and only two double-lined eclipsing binaries with a Bp component, namely AO Vel (=HD 68826) and HD 123335, are known to date (e.g., Clausen et al. 1995, from here on $\mathrm{CGvH}$; Hensberge et al. 2004). In this paper, we perform a spectroscopic analysis of the eclipsing SB2 system $\mathrm{AO}$ Vel consisting of two late B-type stars with a BpSi primary component (Bidelman \& MacConnell 1973), i.e. of a type generally displaying rather large spectroscopic variations, so that one can infer that there are significant surface inhomogeneities. Numerous four-colour photometric observations have been obtained from 1974 to 1991 with the Danish $50 \mathrm{~cm}$ telescope at La Silla $(\mathrm{CGvH})$. Times of mid-eclipse for a total of 16 primary and 10 secondary minima have been determined from all available photoelectric observations and photometric elements and apsidal motion parameters have been determined from complete $u v b y$ light curves. This study revealed the presence of a third component in a wide orbit with an orbital period of $25.6 \mathrm{yr}$. The orbital period of the eclipsing system appeared 
to be 1.58 days and the orbit was found only slightly eccentric, with a quite fast apsidal motion $(U=56.8 \mathrm{yr})$. Very recently, Wolf \& Zejda (2005) published seven additional times of minima obtained from the ASAS-3 database (Pojmanski 2002) and new photoelectric $U B V$ observations. As a result, they have improved the apsidal motion parameters and the orbit of the third body, for which they found a period of $33 \mathrm{yr}$.

In spite of the considerable amount of observational data collected for decades, absolute dimensions of the components remained unknown since no accurate radial velocity observations had ever been performed for this system.

\section{Observations and spectroscopic analysis}

\subsection{Observations}

In March 2004 we obtained five spectra of AO Vel with a typical S/N of about 100-150 over three nights with the echelle spectrograph FEROS at the $2.2 \mathrm{~m}$ telescope at La Silla, to obtain radial velocities and, ultimately, the individual masses of the components. Their wavelength range is 3530-9220 , and the nominal resolving power is 48000 . To our surprise, we found that the spectral line profile in the FEROS spectra exhibit a variable and complex structure indicating four spectral companions at some phases. In order to confirm the identification of the four companions and to derive their respective temporal behavior, we obtained six additional spectra in January 2005 with the $2.1 \mathrm{~m}$ telescope of the CASLEO and the echelle spectrograph REOSC. These spectra cover the range 3600-6000 $\AA$ at a resolution of 13000 and a typical $\mathrm{S} / \mathrm{N}$ of about 90 . A careful inspection of the spectral line morphology, along with the photometric ephemeris published by $\mathrm{CGvH}$, allowed us to identify in the spectral line profiles four stellar components forming two short-period double-lined spectroscopic binaries. In the following, we identify these stars as A, B, C and D. The two more massive stars form the eclipsing binary $(A+B)$ with an orbital period of 1.58 days, the primary component being the BpSi star. The other spectroscopic binary $(\mathrm{C}+\mathrm{D})$ has a period of 4.15 days, and its components have a similar spectral type B9-A0.

\subsection{Spectra separation and RV measurement}

In order to obtain separate spectra for all four components of the system and to measure their radial velocities (RVs), we applied the iterative method described by González \& Levato (2006), which was adapted here for multiple systems. This algorithm computes the spectra of the individual components and the RVs iteratively. In each step the computed spectra are used to remove the spectral features of all but one component from the observed spectra. The resulting single-lined spectra are used to measure the RV of that component and to compute its spectrum by combining them appropriately. Starting values for RVs were measured in the FEROS spectra by crosscorrelation against spectra of slowly rotating stars of similar spectral type, HD 175640 and HD 196426, which had been observed with FEROS in May 2000. The RV of these templates were determined by measuring individual spectral lines. From these preliminary RVs we calculated the spectra of the four individual components using our high resolution FEROS spectra. For these calculations we considered only four of our five FEROS spectra, since in the first one, taken at MJD 53070.013, three components appear strongly blended, making the determinations of RVs less reliable. The CASLEO spectra also were not considered because of their lower resolution. During the computation of the spectra of the individual components, the RVs in those four FEROS spectra were redetermined.

Once the spectra of the four stellar components were obtained, they were used to compute the RVs from all our $11 \mathrm{ob}-$ served spectra. Figure 1 shows the RVs of the four stars as a function of time; the left panel shows the multiple morphology of the cross-correlation function.

Table 1 presents the measured RVs for all four stellar components. Early observations (before MJD 53373) are those of FEROS. The assigned RV errors are formal ones calculated within the cross-correlation analysis. The quality of the $\mathrm{RV}$ measurements is also influenced by the uncertainty in the computed spectra, which are used to remove the lines of the remaining components from the observed spectra. In order to evaluate this contribution to the RVs errors, we calculated the RVs using different subsamples of observed spectra and in most cases we found that RV differences are comparable with their formal errors. However, in the spectrum taken on MJD 53070.0187 the RVs of components A, B and C are not well defined. The RV computations converge poorly and depend strongly on the initial velocities. In this case we assigned larger errors so that all possible solutions are included.

\subsection{Spectral analysis}

To normalize the spectra of individual stellar components we scaled the combined continuum by their relative luminosity. We used the relative fluxes in $y$ and $b$ photometric bands provided by $\mathrm{CGvH}$ for the star $\mathrm{A}$ and the star $\mathrm{B}$, and the combined flux of $\mathrm{C}+\mathrm{D}$. Assuming that the fluxes of stars $\mathrm{C}$ and $\mathrm{D}$ are equal, we obtain relative contributions to the observed continuum of $0.40,0.28,0.16$, and 0.16 for components $\mathrm{A}, \mathrm{B}, \mathrm{C}$ and $\mathrm{D}$, respectively. The uncertainty of these scaling factors is about $5 \%$, and should be considered as a possible systematic error in the intensity of spectral lines.

For each component in the system, we calculated synthetic spectra using the SYNTH code (Piskunov 1992), the VALD database (Kupka et al. 1999) and ATLAS9 model atmospheres (Kurucz 1993). Starting from models with different atmosphere parameters, we compared a variety of syntheses with observed spectra to determine the best fits (Table 2). Because of the rather poor quality of the spectra, especially of the faint components $\mathrm{C}$ and $\mathrm{D}$, we mainly took into account the region 4400-4600 $\AA$ which includes the He I 4471 and Mg II 4481 lines, as well as the 5000-5100 and 6300-6400 $\AA$ regions with some prominent $\mathrm{Si}-$ and Fe-lines. The estimated errors in $T_{\text {eff }}$ are of the order of $\pm 250 \mathrm{~K}$ for component $\mathrm{B}$ and $\pm 500 \mathrm{~K}$ for components $\mathrm{C}$ and $\mathrm{D}$. The best choice of microturbulence velocity, $v_{\text {mic }}$, was found to be $0 \mathrm{~km} \mathrm{~s}^{-1}$ for $\mathrm{A}$ and $\mathrm{B}$ and $2 \mathrm{~km} \mathrm{~s}^{-1}$ for $\mathrm{C}$ and $\mathrm{D}$. 

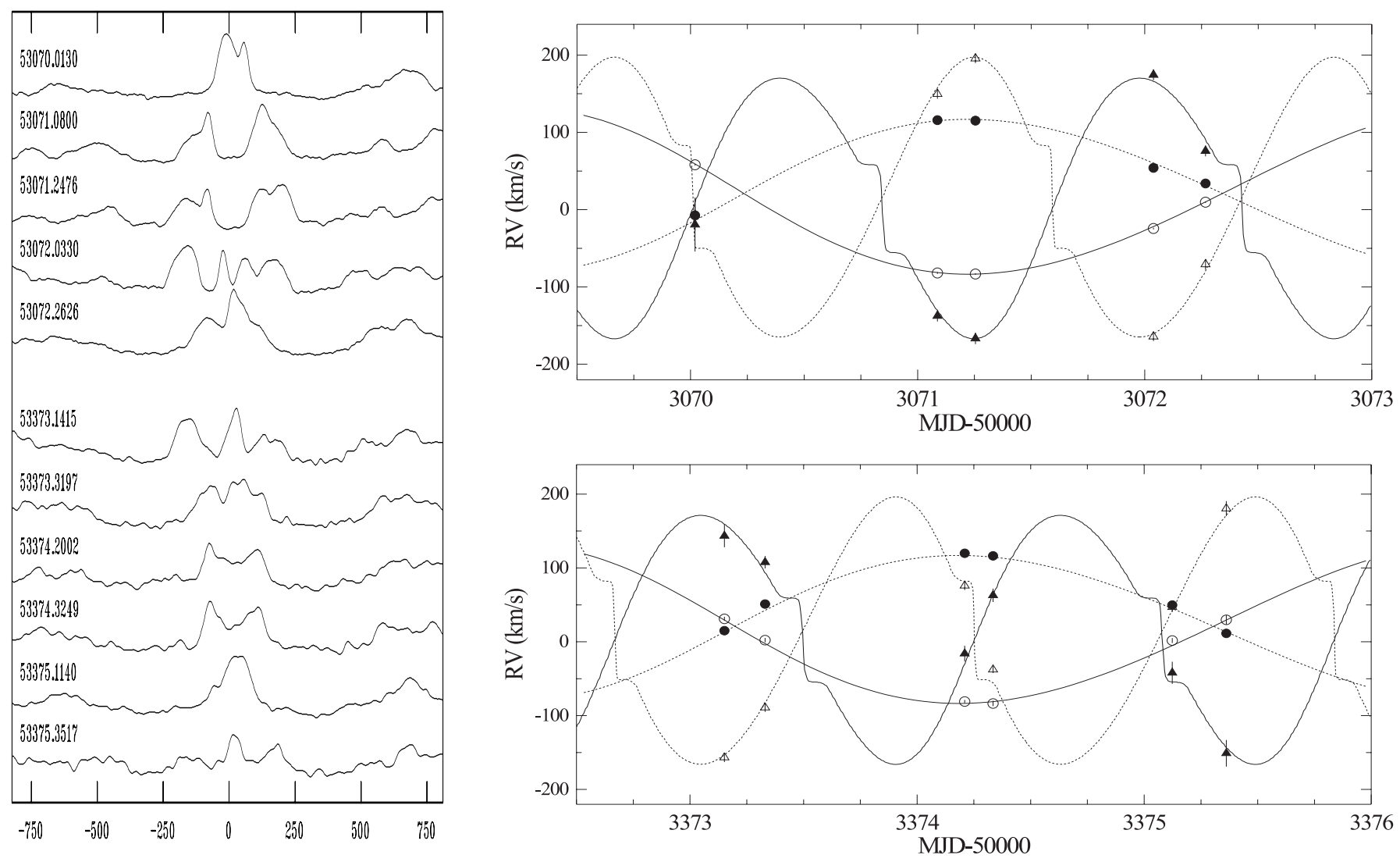

Fig. 1. Cross-correlation function (left) and RV curves (right) for FEROS (top) and CASLEO (bottom) spectra. Filled triangles, open triangles, filled circles and open circles represent stars A, B, C and D, respectively.

Table 1. Radial velocities for all four companions.

\begin{tabular}{crrrrrrrr}
\hline \hline MJD & $\begin{array}{c}R V_{\mathrm{A}} \\
\mathrm{km} \mathrm{s}^{-1}\end{array}$ & $\begin{array}{c}\epsilon_{\mathrm{A}} \\
\mathrm{km} \mathrm{s}^{-1}\end{array}$ & $\begin{array}{c}R V_{\mathrm{B}} \\
\mathrm{km} \mathrm{s}^{-1}\end{array}$ & $\begin{array}{c}\epsilon_{\mathrm{B}} \\
\mathrm{km} \mathrm{s}^{-1}\end{array}$ & $\begin{array}{c}R V_{\mathrm{C}} \\
\mathrm{km} \mathrm{s}^{-1}\end{array}$ & $\begin{array}{c}\epsilon_{\mathrm{C}} \\
\mathrm{km} \mathrm{s}^{-1}\end{array}$ & $\begin{array}{c}R V_{\mathrm{D}} \\
\mathrm{km} \mathrm{s}^{-1}\end{array}$ & $\begin{array}{c}\epsilon_{\mathrm{D}} \\
\mathrm{km} \mathrm{s}^{-1}\end{array}$ \\
\hline 53070.0187 & -19 & 35 & -7 & 20 & -7.3 & 15.0 & 59.8 & 1.0 \\
53071.0875 & -137 & 7 & 150 & 6 & 115.9 & 2.3 & -81.9 & 1.1 \\
53071.2551 & -167 & 7 & 196 & 6 & 115.3 & 2.3 & -83.2 & 1.0 \\
53072.0387 & 174 & 7 & -164 & 6 & 54.2 & 2.2 & -24.3 & 1.1 \\
53072.2683 & 76 & 7 & -71 & 7 & 34.1 & 2.1 & 9.9 & 1.0 \\
53373.1513 & 143 & 15 & -157 & 6 & 15.2 & 4.3 & 31.2 & 2.8 \\
53373.3296 & 108 & 8 & -89 & 6 & 51.2 & 3.7 & 2.3 & 2.9 \\
53374.3348 & 63 & 9 & -38 & 5 & 116.5 & 2.9 & -83.5 & 3.6 \\
53374.2101 & -16 & 11 & 76 & 6 & 120.0 & 3.9 & -80.9 & 2.2 \\
53375.1239 & -42 & 15 & 47 & 6 & 49.3 & 4.1 & 1.8 & 3.3 \\
53375.3616 & -151 & 18 & 180 & 10 & 11.5 & 2.0 & 29.9 & 5.9 \\
\hline
\end{tabular}

Since the deeper eclipse in the primary system, $\mathrm{A}+\mathrm{B}$, corresponds to the occultation of star A, this star is not only the larger and more massive one, but also the hotter one. However, He I lines are weaker than in star B, while Si II lines are very strong. Since the component A is a chemically peculiar star, it is not possible to derive its temperature by simply comparing observations with a variety of solar composition models of different $T_{\text {eff }}$ and $\log g$ values. A detailed multi-element abundance analysis would be needed to determine accurate atmospheric parameters. However, such an analysis is not possible yet because of the unavailability of high-resolution and high signal-to noise spectra. For this reason we adopted for the 

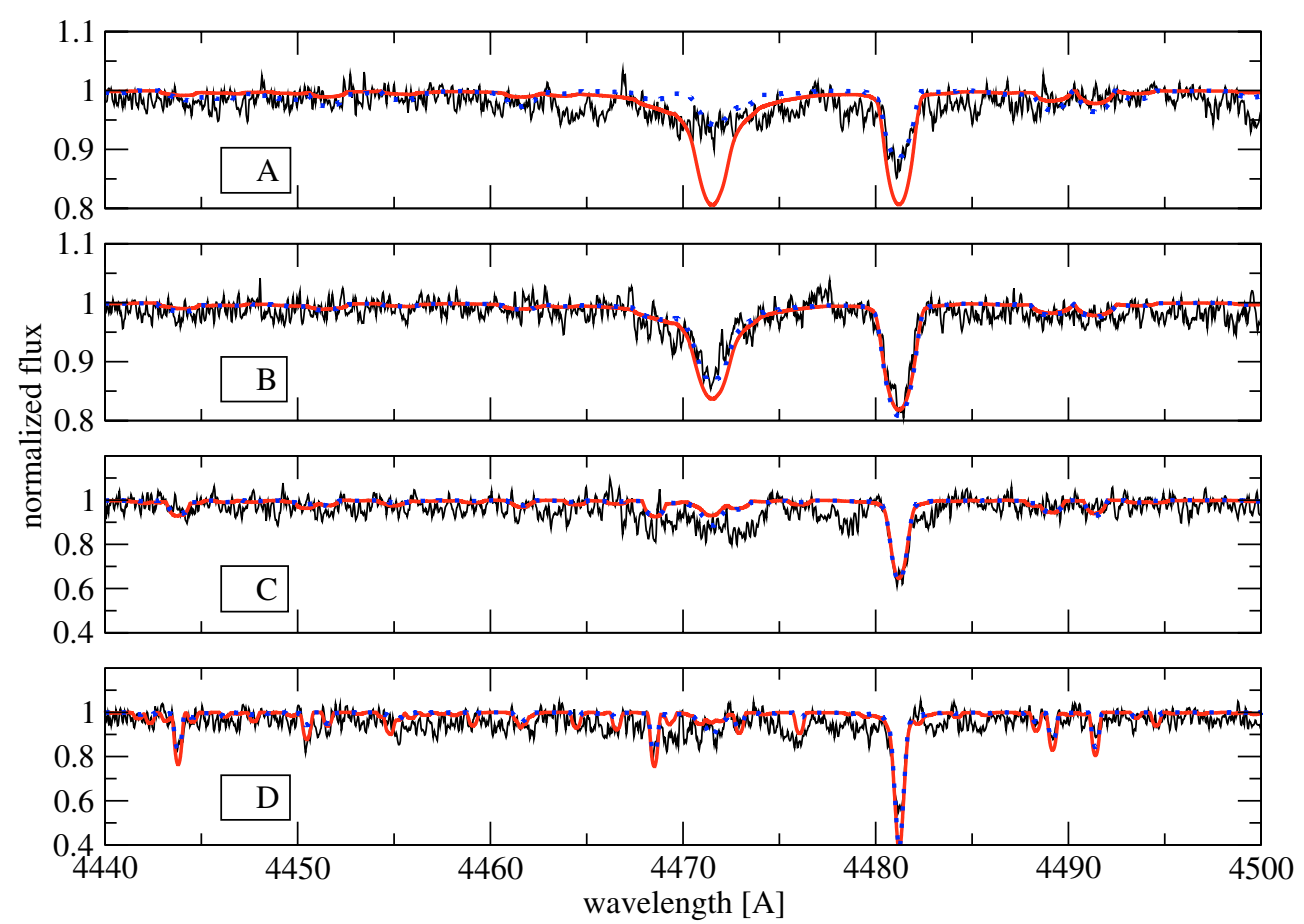

Fig. 2. Observed spectra of components A, B, C, and D (thin lines) compared with synthetic spectra (solid and dotted lines). Atmospheric parameters for modeled spectra are as following. A) Solid line: synthetic spectrum with parameters from stellar models $\left(T_{\text {eff }}=13700 \mathrm{~K}\right.$, $\log g=4.3$ ) and solar composition, dotted line: same model but with $\mathrm{Mg}$ and He abundances decreased by 1.45 and 1.04 dex; B) solid line: synthetic spectrum with parameters from stellar models $\left(T_{\text {eff }}=13200 \mathrm{~K}, \log g=4.3\right)$, dotted line: best fit for $\mathrm{Mg} / \mathrm{He}$ ratio and metal lines $\left(T_{\text {eff }}=12500 \mathrm{~K}, \log g=4.3\right)$; C) solid line: $T_{\text {eff,min }}=10000 \mathrm{~K}, \log g=4.1$, dotted line: $T_{\text {eff,max }}=11000 \mathrm{~K}, \log g=4.1$; D) solid line: $T_{\text {eff, } \min }=9500 \mathrm{~K}, \log g=4.2$, dotted line: $T_{\text {eff, } \max }=10500 \mathrm{~K}, \log g=4.2$.

Table 2. Atmospheric parameters derived from comparison with synthetic spectra.

\begin{tabular}{cccc}
\hline \hline Star & $\begin{array}{c}T_{\text {eff }} \\
{[\mathrm{K}]}\end{array}$ & $\log g$ & $\begin{array}{c}v \sin i \\
{\left[\mathrm{~km} \mathrm{~s}^{-1}\right]}\end{array}$ \\
\hline A & $13700^{a}$ & 4.3 & 65 \\
B & $12500 \pm 250 \mathrm{~K}$ & $4.3 \pm 0.1$ & 73 \\
C & $10500 \pm 500 \mathrm{~K}$ & $4.1 \pm 0.2$ & 40 \\
D & $10000 \pm 500 \mathrm{~K}$ & $4.2 \pm 0.2$ & 18 \\
\hline
\end{tabular}

${ }^{a}$ Adopted from stellar models according to the observed mass and radius.

component A the effective temperature predicted by stellar models for a normal star of the same mass and radius. Using the Geneva stellar evolution models (Schaller et al. 1992) and the mass and radius calculated from the light and RV curves (see next section) we found for the A component $T_{\text {eff }}=13700$. In Fig. 2 we show the observed spectrum (thin line) together with the synthetic spectrum computed with the atmosphere parameters $T_{\text {eff }}=13700, \log g=4.3$ and solar element abundances (solid line). In order to reproduce the He I and $\mathrm{Mg}$ II features in the 4470-4480 $\AA$ region by the synthesis using the same atmosphere parameters the abundances of $\mathrm{He}$ and $\mathrm{Mg}$ had to be decreased by 1.45 and 1.04 dex, respectively (dotted line). From the Si II lines in the spectrum of star A we derived a $\mathrm{Si}$-overabundance of $\approx 0.5$ dex relative to the solar composition model, which is in good agreement with the classification of this star as BpSi by Bidelman \& MacConnell (1973). Star B is a B8 V normal star (Fig. 2). The observed spectrum can be very well fitted by a synthetic spectrum assuming solar composition, $T_{\text {eff }}=12500 \pm 250 \mathrm{~K}$ and $\log g=4.3 \pm 0.1$.

The spectra of stars belonging to the spectroscopic binary $\mathrm{C}+\mathrm{D}$ are similar to each other and correspond approximately to spectral type B9-A0. The spectral analysis of the system $\mathrm{C}+\mathrm{D}$ using the SYNTH code indicates $T_{\text {eff }}=10500 \pm 500 \mathrm{~K}$ for the star C (Fig. 2), which is the more massive companion, and $10000 \pm 500 \mathrm{~K}$ for the less massive companion D (Fig. 2). The He I line in the spectra of $\mathrm{C}$ and $\mathrm{D}$ cannot be considered as a reliable temperature indicator since the continuum determination in this region is rather uncertain. The uncertainty of our temperature estimates, which are based only on a few well resolved, unblended Fe lines, is rather high. The majority of $\mathrm{Mg}$ and $\mathrm{Fe}$ lines in the spectrum of star $\mathrm{C}$ can be modelled rather well assuming a purely solar composition, $T_{\text {eff }}=10500 \mathrm{~K}$ and $\log g=4.1 \pm 0.2$. While most metal lines in the spectrum of star D can be well reproduced by the synthesis assuming solar composition, $T_{\text {eff }}=10000 \mathrm{~K}$ and $\log g=4.2 \pm 0.2$, the observed $\mathrm{Mg}$ II line at $4481 \AA$ indicates a lower abundance $(\approx-0.4 \mathrm{dex})$ than predicted by the model.

For a more careful spectral analysis a larger number of observed spectra at different orbital phases is needed, not only to improve the signal-to-noise ratio but also to assure a better disentangling of spectral components. 


\section{Orbital analysis}

\subsection{Spectroscopic orbits}

Spectroscopic orbits for the two binaries $A+B$ and $C+D$ were calculated using the $11 \mathrm{RV}$ measurements of each star. Weights were given to the RV measurements according to their uncertainties.

For the system $\mathrm{C}+\mathrm{D}$ we used the least squares method to determine the following parameters: $P_{\mathrm{CD}}$ (orbital period), $K_{\mathrm{C}}$, $K_{\mathrm{D}}$ (RV amplitudes), $V \gamma_{\mathrm{CD}}$ (center-of-mass RV), $e_{\mathrm{CD}}$ (eccentricity), $\omega_{\mathrm{CD}}$ (argument of the periastron), and $T_{\mathrm{CD}}$ (time of periastron passage). The rms of the residuals is $4.9 \mathrm{~km} \mathrm{~s}^{-1}$ for star C, while for star D it is $1.0 \mathrm{~km} \mathrm{~s}^{-1}$ for La Silla observations and $4.1 \mathrm{~km} \mathrm{~s}^{-1}$ for CASLEO spectra. These values are consistent with the measurement errors.

In the case of the system $\mathrm{A}+\mathrm{B}$, we fitted the RV curves using the Wilson \& Devinney program (Wilson \& Devinney 1971; Wilson 1990) in order to take into account the proximity effects which are significant in such a short-period binary. We lowered the weight of observations taken during the eclipses by a factor of 2, since the effect of partial eclipse on the RVs (Rossiter effect, Rossiter 1924) has not been specifically modelled during our calculation of the component spectra and RV measurements.

A detailed study of the spectral line profile variability during the eclipses would be especially interesting to obtain information about surface inhomogeneities of chemical elements in the BpSi primary. However, a larger number of high-resolution spectra are needed for such a study, which is thus deferred to a future work.

Reasonable values for the atmospheric parameters required by the Wilson \& Devinney program were assigned according to the spectral types. We adopted the relative radii and orbital inclination calculated by $\mathrm{CGvH}$ from the analysis of the photoelectric $u v b y$ light curves of Grønbech (1987). We let the epoch $T_{\mathrm{AB}}$ as a free parameter since the photometric ephemeris calculated by $\mathrm{CGvH}$ were not compatible with our RV curves. As described in the next section, this and other inconsistencies led us to reanalyze the photometric ephemeris and to propose parameters of the long-period orbit that are quite different from those found by previous works. The orbital parameters resulting from the RV curve analysis are listed in Table 3.

\subsection{The long-period orbit between the two binaries}

To better constrain the fundamental parameters of the AO Vel system, we did an in-depth revision of all published data and analyzed all available times of minima of the eclipsing binary. In the study of the eclipsing system $\mathrm{A}+\mathrm{B}, \mathrm{CGvH}$ fitted the epochs of 26 eclipses measured throughout 26 years using a period of $1.584653 \pm 0.000005$ days and considering the combined effect of the apsidal motion of the orbit at a rate of $0.0275 \%$ cycle and the light time effect due to the orbit with a third body (our binary system $\mathrm{C}+\mathrm{D}$ ). However, the time of minimum predicted for the epoch of our observations differs from our spectroscopic ephemeris by $0.060 \pm 0.026$ days. In addition, according to the "third body" orbit found by $\mathrm{CGvH}$
Table 3. Spectroscopic orbits of binary systems $\mathrm{C}+\mathrm{D}$ and $\mathrm{A}+\mathrm{B}$.

\begin{tabular}{|c|c|c|}
\hline Parameter & Units & Value \\
\hline$P_{\mathrm{CD}}$ & days & $4.15008 \pm 0.00016$ \\
\hline$T_{\mathrm{CD}}$ (periastron) & MJD & $53074.059 \pm 0.060$ \\
\hline$V \gamma_{\mathrm{CD}}$ & $\mathrm{km} \mathrm{s}^{-1}$ & $21.2 \pm 0.7$ \\
\hline$K_{\mathrm{C}}$ & $\mathrm{km} \mathrm{s}^{-1}$ & $98.3 \pm 2.1$ \\
\hline$K_{\mathrm{D}}$ & $\mathrm{km} \mathrm{s}^{-1}$ & $107.4 \pm 1.3$ \\
\hline$\omega_{\mathrm{CD}}$ & deg & $60 \pm 6$ \\
\hline$e_{\mathrm{CD}}$ & & $0.047 \pm 0.015$ \\
\hline$a_{\mathrm{CD}} \sin i_{\mathrm{CD}}$ & $R_{\odot}$ & $16.84 \pm 0.23$ \\
\hline$q_{\mathrm{CD}}$ & & $0.916 \pm 0.024$ \\
\hline$M_{\mathrm{C}} \sin ^{3} i_{\mathrm{CD}}$ & $M_{\odot}$ & $1.94 \pm 0.07$ \\
\hline$M_{\mathrm{D}} \sin ^{3} i_{\mathrm{CD}}$ & $M_{\odot}$ & $1.77 \pm 0.08$ \\
\hline$P_{\mathrm{AB}}$ & days & $1.584584^{\mathrm{a}}$ \\
\hline$T_{\mathrm{AB}}$ (periastron) & MJD & $53069.8821 \pm 0.0055$ \\
\hline$T_{\mathrm{AB}}(\operatorname{Min} \mathrm{I})$ & MJD & $53070.8440 \pm 0.0055$ \\
\hline$V \gamma_{\mathrm{AB}}$ & $\mathrm{km} \mathrm{s}^{-1}$ & $8.9 \pm 2.3$ \\
\hline$q_{\mathrm{AB}}$ & & $0.931 \pm 0.033$ \\
\hline$\omega_{\mathrm{AB}}$ & deg & $236.5^{a}$ \\
\hline$\dot{\omega}$ & $\operatorname{deg} \mathrm{yr}^{-1}$ & $0.0174^{a}$ \\
\hline$e_{\mathrm{AB}}$ & & $0.0741^{a}$ \\
\hline$i_{\mathrm{AB}}$ & deg & $88.5^{b}$ \\
\hline$M_{\mathrm{A}}$ & $M_{\odot}$ & $3.63 \pm 0.18$ \\
\hline$M_{\mathrm{B}}$ & $M_{\odot}$ & $3.38 \pm 0.18$ \\
\hline$a_{\mathrm{AB}}$ & $R_{\odot}$ & $10.94 \pm 0.19$ \\
\hline$R_{\mathrm{A}}$ & $R_{\odot}$ & $2.34^{c} \pm 0.08$ \\
\hline$R_{\mathrm{B}}$ & $R_{\odot}$ & $2.11^{c} \pm 0.08$ \\
\hline
\end{tabular}

${ }^{a}$ Calculated from photometric ephemeris analysis (Sect. 3.2). $P_{\mathrm{AB}}$ and $\omega_{\mathrm{AB}}$ are calculated for MJD 53070.

${ }^{b}$ Adopted from CGvH.

${ }^{c}$ Computed using the photometric relative radii published by $\mathrm{CGvH}$.

for the time of our FEROS observations the barycentric RV of the binary $\mathrm{A}+\mathrm{B}$ should be higher than that of the binary $\mathrm{C}+\mathrm{D}$ for La Silla and CASLEO observations, which is absolutely incompatible with the observed $\mathrm{RVs}$ since, according to our measurements, $V \gamma_{\mathrm{AB}}-V \gamma_{\mathrm{CD}}=-12.3 \pm 2.4 \mathrm{~km} \mathrm{~s}^{-1}$. Finally, the third body orbit of $\mathrm{CGvH}$ is unable to match the 38 old photographic observations taken by Oosterhoff \& van Houten (1949) between the years 1902 and 1935. CGvH have admitted this disagreement in their paper and speculated that might be a consequence of some kind of longer time scale period variation.

On the other hand, the parameters recently published by Wolf \& Zejda (2005) for the wide orbit are very different from those of CGvH. The ephemeris calculated by Wolf \& Zejda (2005) agree with our observations within 0.003 days. They found an eccentric orbit ( $e=0.21$ ) with a period of $33.3 \mathrm{yr}$, which might account for the observed RV difference between the two binaries. However, it is also in disagreement with the old photographic data.

For these reasons, we decided to recompute the apsidal motion and the third body orbit using all the available times of 


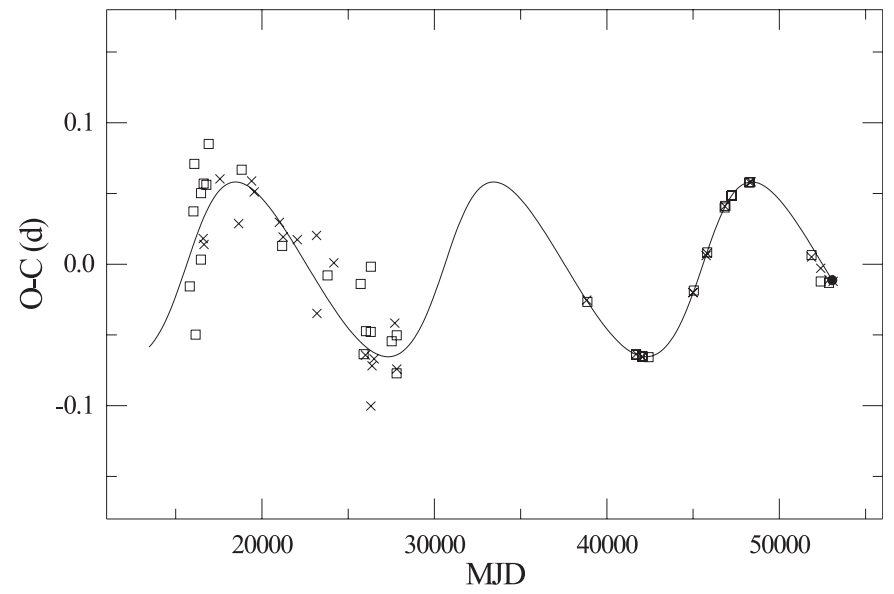

Fig. 3. Times of minimum shifts caused by the third body orbit. Symbols are the differences between the observed times of minima and the times of minima calculated using mean ephemeris, after correction for apsidal motion (primary eclipses are marked by squares, secondary eclipses are marked by crosses and the filled circle shows Min I calculated by fitting our RV curves).

minimum along with our spectroscopic observations. In view of the very different quality of the various photometric sources, we have given weight 1 to the photoelectric minima (26 from CGvH and one from Wolf \& Zejda 2005), weight 0.1 to the ASAS-3 data, and weight 0.01 to the much less precise photographic data. We have also included the time of minimum predicted by our spectroscopic orbit as a single point with unit weight.

The key difference with respect to the $\mathrm{CGvH}$ calculations is that we do not assume the wide orbit to be circular. On the other hand, we have included the photographic data, which were not considered by Wolf \& Zejda (2005). Consequently, we were able to find a solution that is compatible with both our spectroscopic orbit and the old photographic observations. Figure 6 shows our fit of the times of minima variation due to the 3rd body (cf. with Fig. 4 in CGvH and Fig. 6 in Wolf \& Zejda 2005). The RMS of the residuals was $4 \times 10^{-4}$ days for the photoelectric minima, $7 \times 10^{-3}$ days for the ASAS-3 photometry and $2.6 \times 10^{-2}$ days for the photographic data.

In addition to the determination of the parameters of the orbit $\mathrm{AB}+\mathrm{CD}$, the ephemeris fitting provided simultaneously the orbital period of the eclipsing binary and its apsidal motion rate. Table 4 shows the results obtained. The parameters we found for the apsidal motion are indistinguishable from those found by CGvH and Wolf \& Zejda (2005). However, the parameters defining the orbit $\mathrm{AB}+\mathrm{CD}$ are essentially different from those found by $\mathrm{CGvH}\left(P_{\mathrm{o}}=25.6 \pm 2.5 \mathrm{yr}\right.$; $a_{0,1} \sin i_{\mathrm{o}}=0.041 \pm 0.006$ days) or by Wolf \& Zejda (2005) $\left(P_{\mathrm{o}}=33.3 \pm 0.5 \mathrm{yr} ; a_{\mathrm{o}, 1} \sin i_{\mathrm{o}}=0.052 \pm 0.004\right.$ days $)$, and also the binary period $P_{\mathrm{AB}}$ differs significantly.

With the new parameters for the wide orbit the eclipsing binary is at present moving toward the observer, as expected according to the observed RVs. The predicted RV for the eclipsing binary is $-6.0 \mathrm{~km} \mathrm{~s}^{-1}$ with respect to the center-of-mass of the quadruple system, which is consistent with the observed velocities (see Sect. 4.1). However, more accurate RVs (errors of
Table 4. Orbital parameters derived from the analysis of times of minima.

\begin{tabular}{lcc}
\hline \hline Parameter & Units & Value \\
\hline \multicolumn{3}{c}{ Orbit $\mathrm{A}+\mathrm{B}$} \\
\hline$P_{\mathrm{AB}}$ & days & $1.5846154 \pm 0.000002$ \\
$T_{\mathrm{AB}}($ MinI $)$ & $\mathrm{MJD}$ & $45043.6608 \pm 0.0004$ \\
$P_{\text {aps. motion }}$ & $\mathrm{yr}$ & $54.72 \pm 0.45$ \\
$T_{\omega=0}$ & $\mathrm{MJD}$ & $39490 \pm 90$ \\
$e_{\mathrm{AB}}$ & \multicolumn{2}{c}{$0.0741 \pm 0.0004$} \\
\hline \multicolumn{3}{c}{ Orbit AB $+\mathrm{CD}$} \\
\hline$P_{\mathrm{o}}$ & $\mathrm{yr}$ & $41.0 \pm 0.2$ \\
$T_{\mathrm{o}}($ periastron $)$ & $\mathrm{MJD}$ & $45839 \pm 11$ \\
$T_{\mathrm{o}}($ conjunction $)$ & $\mathrm{MJD}$ & $47803 \pm 120$ \\
$a_{\mathrm{o}, 1} \sin i_{\mathrm{o}}$ & days & $0.0642 \pm 0.0004$ \\
$a_{\mathrm{o}, 1} \sin i_{\mathrm{o}}$ & $\mathrm{AU}$ & $11.15 \pm 0.07$ \\
$e_{\mathrm{o}}$ & \multicolumn{2}{c}{$0.291 \pm 0.005$} \\
$\omega_{\mathrm{o}}$ & deg & $11.8 \pm 1.4$ \\
\hline
\end{tabular}

about $0.3-0.7 \mathrm{~km} \mathrm{~s}^{-1}$ in $\left.V \gamma_{\mathrm{AB}}-V \gamma_{\mathrm{CD}}\right)$ would be required for the mass-ratio $M_{\mathrm{C}+\mathrm{D}} / M_{\mathrm{A}+\mathrm{B}}$ to be calculated with reasonable precision $(5-10 \%)$.

\section{Physical parameters}

\subsection{Stellar parameters and evolutionary state}

For the eclipsing pair we have derived absolute masses and dimensions using the photometric elements (inclination and relative radii) published by $\mathrm{CGvH}$ along with our spectroscopic data. In the mass-radius diagram shown in Fig. 4 both A and B are located near the ZAMS. For comparison, isochrones of the Geneva stellar models (Schaller et al. 1992) for solar composition are also plotted in Fig. 4. The mass and radius of star A indicates that the age of the system is less than about $5 \times 10^{7} \mathrm{yr}$, being compatible with the ZAMS. This corresponds to less than $25 \%$ of the main sequence lifetime for star A.

Since the orbital inclination for the binary $\mathrm{C}+\mathrm{D}$ is unknown, the individual masses of these stars cannot be derived from the RV curves. However, some information can be obtained from the outer orbit between the two systems and the mass of the binary A+B. In the following we use subindex "o" for the parameters of the orbit binding both binary systems. In particular, $M_{\mathrm{o}}$ is the total mass of the quadruple system and $q_{\mathrm{o}}=M_{\mathrm{C}+\mathrm{D}} / M_{\mathrm{A}+\mathrm{B}}$.

From the ephemeris analysis of the eclipsing binary we have found the parameter $a_{\mathrm{0}, 1} \sin i_{\mathrm{o}}$, which is related to the semiaxis of the relative orbit $\left(a_{0}\right)$ through:

$a_{\mathrm{o}} \sin i_{\mathrm{o}}=a_{\mathrm{o}, 1} \sin i_{\mathrm{o}} \cdot\left(1+q_{\mathrm{o}}^{-1}\right)$.

Combining this expression with the third Kepler equation

$M_{\mathrm{o}}=M_{\mathrm{A}+\mathrm{B}} \cdot\left(1+q_{\mathrm{o}}\right)=a_{\mathrm{o}}^{3} \cdot P_{\mathrm{o}}^{-2}$,

we obtain:

$\frac{\left(1+q_{\mathrm{o}}\right)^{2}}{q_{\mathrm{o}}^{3}}=\left(a_{\mathrm{o}, 1} \sin i_{\mathrm{o}}\right)^{-3} \cdot P_{\mathrm{o}}^{2} \cdot M_{\mathrm{A}+\mathrm{B}} \cdot \sin ^{3} i_{\mathrm{o}}$, 


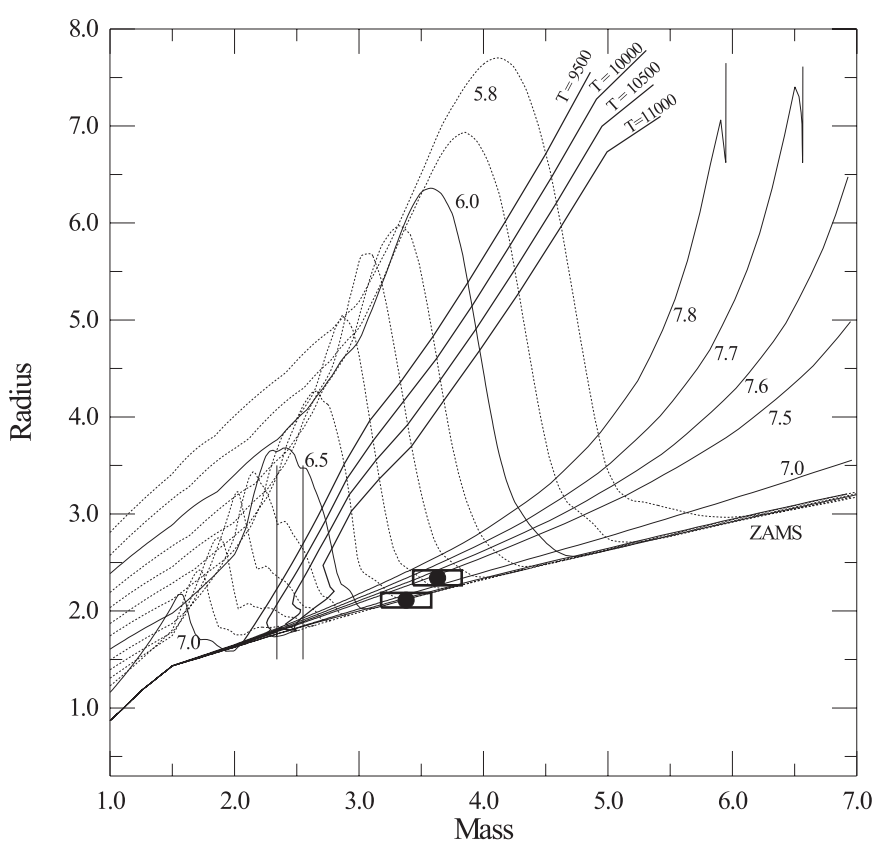

Fig. 4. Mass-radius diagram. Filled circles with error boxes are stars A and $\mathrm{B}$ belonging to the eclipsing system. Minimum masses for stars $\mathrm{C}$ and $\mathrm{D}$ are marked with vertical bars. Main sequence (Schaller et al. 1992, continuous lines) and pre-main sequence (Bernasconi 1996, dotted lines) isochrones are plotted and labeled with $\log ($ age). Thick lines are isotherms interpolated for pre-main-sequence models.

where $\left(a_{\mathrm{o}, 1} \sin i_{\mathrm{o}}\right)$ is given in $\mathrm{AU}$ and $P_{\mathrm{o}}$ in years. For a given value of $i_{\mathrm{o}}$ this equation provides $q_{\mathrm{o}}$, and hence the absolute masses of the system $\mathrm{C}+\mathrm{D}: M_{\mathrm{D}}=q_{\mathrm{o}} \cdot M_{\mathrm{A}+\mathrm{B}} /\left(q_{\mathrm{CD}}+1\right), M_{\mathrm{C}}=$ $q_{\mathrm{CD}} \cdot M_{\mathrm{D}}$. From the condition $i_{\mathrm{o}} \leq 90^{\circ}$ a lower limit for the masses of components $\mathrm{C}$ and $\mathrm{D}$ can be established. The resulting minimum masses are $2.55 \pm 0.09 M_{\odot}$ and $2.34 \pm 0.10 M_{\odot}$.

Using these minimum masses for the stars $\mathrm{C}$ and $\mathrm{D}$ and their spectroscopic orbit, we obtain the orbital inclination of the non-eclipsing binary to be $i_{\mathrm{CD}} \leq 66.0 \pm 2.4 \mathrm{deg}$. If the mass of $\mathrm{C}+\mathrm{D}$ is close to their lower limit, then $q_{\mathrm{o}} \approx 0.70$, and the expected RV difference between the two binaries is $V \gamma_{\mathrm{AB}}-$ $V \gamma_{\mathrm{CD}}=-14.5 \pm 0.6 \mathrm{~km} \mathrm{~s}^{-1}$, in agreement with the observed value $\left(-12.3 \pm 2.4 \mathrm{~km} \mathrm{~s}^{-1}\right)$.

The parameters of the wide orbit restrict the position of stars $\mathrm{C}$ and $\mathrm{D}$ in the mass-radius diagram to the right of the two continuous vertical lines in Fig. 4.

Assuming the four stars to be coeval, stars C and D would be very close to the ZAMS, considering the youth of the more massive stars. Even the possibility of them being pre-main sequence stars must be considered. In Fig. 4 pre-main sequence isochrones for solar composition from Bernasconi (1996) are plotted. The spectroscopic temperatures and the luminosity difference between the two binary systems provide useful constraints on the evolutionary state of the stars C and D. Four isotherms have been interpolated in the pre-main sequence models of Bernasconi (1996) at temperatures 9500, 10000, 10500 , and $11000 \mathrm{~K}$. These curves define in Fig. 4 two overlapping strips corresponding to stellar models with temperatures close to those inferred from the spectra of components $\mathrm{C}$ and D (9500-10500 K and 10000-11000 K, respectively).

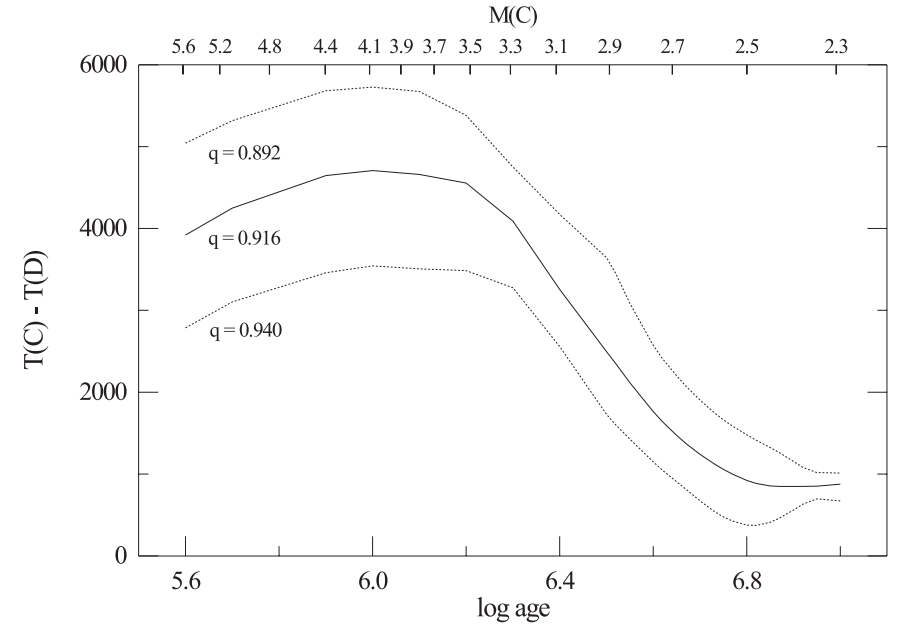

Fig. 5. Expected temperature difference of stars $C$ and $D$ as a function of age, according to pre-main sequence models of Bernasconi (1996).

Within these strips, the younger massive models are not compatible with the observations for various reasons. First of all, if all four stars are assumed to be coeval, pre-main sequence ages of the order or below $10^{6.3} \mathrm{yr}$ should be discarded because of the small radius of star B. Second, massive models for stars C and D would be too luminous with respect to components $\mathrm{A}$ and $\mathrm{B}$. More precisely, models with log (age) $<6.55$ are not compatible with the light-ratio derived from the light curve analysis by $\mathrm{CGvH}$. Finally, in the upper portion of the temperature strips in Fig. 4 two stars with a fixed mass-ratio $M_{\mathrm{D}} / M_{\mathrm{C}}=0.916$ would have a temperature difference $T_{\mathrm{C}}-T_{\mathrm{D}}$ larger than expected. This is a consequence of the rapid evolution at larger masses and holds also for the difference in luminosity or radius. As illustration, Fig. 5 shows the temperature difference $T_{\mathrm{C}}-T_{\mathrm{D}}$ as a function of age. Each curve corresponds to the temperature difference of two pre-main sequence stellar models with a given mass ratio (as labeled) and with an average temperature $\left(T_{\mathrm{C}}+T_{\mathrm{D}}\right) / 2=10250 \mathrm{~K}$. Three mass-ratio values have been considered to take into account the observational error of $q_{\mathrm{CD}}$. The similarity of the spectral types of stars $C$ and $D$ favors again the older models. In conclusion, stars $\mathrm{C}$ and $\mathrm{D}$ are most probably on the ZAMS or only slightly younger. The age of the system is not younger than about $10^{6.6} \mathrm{yr}$ in the pre-main sequence grid of Bernasconi (1996).

Additional spectroscopic observations would help to determine the age with higher precision by improving the calculation of stellar parameters for the eclipsing system and the temperature estimates for the less massive binary. In particular, if the pre-main sequence nature of stars C and D was established, then the post-main sequence evolutionary age of the peculiar star A would be less than about $4 \%$ of its main-sequence lifetime.

An additional argument in favor of the youth of the AO Vel system arises from the apsidal motion rate. This rate can be used in combination with the stellar and orbital parameters to derive the average value of the internal structure constant $k_{2}$ of the components. The influence of the system $\mathrm{C}+\mathrm{D}$ on the apsidal motion of the binary $\mathrm{A}+\mathrm{B}$ is negligible due 
to the long period of the outer orbit. Our calculations give $\log k_{2}=-2.25 \pm 0.05$. This is essentially the same value found by $\mathrm{CGvH}$, since this parameter depends strongly on relative radii but only slightly on the masses. Theoretical models for the solar composition (Claret \& Giménez 1992) predict $\log k_{2}=-2.18 \pm 0.02$ for ZAMS stars of the same mass.

\subsection{Absolute magnitude and distance}

Even though absolute masses and dimensions are not precisely known for stars C and D, their contribution to the total luminosity of the quadruple system is rather small, so that the absolute magnitude of the latter can be estimated with reasonable precision. We have derived the absolute visual magnitudes of the components by interpolation in the stellar model grids according to their masses. If all four stars are assumed to be close to the ZAMS the integrated absolute magnitude of the quadruple is $M_{v}=-0.82 \pm 0.12$. This magnitude would be somewhat brighter if the system was more evolved $\left(M_{v}=-0.90\right.$ for $\log \tau=7.5$ ) or if components $C$ and $D$ were assumed to be still PMS stars $\left(M_{v}=-0.95\right.$ for $\left.\log \tau_{\mathrm{PMS}}=6.6\right)$. Using the apparent magnitude $V=9.34$, we obtain an apparent distance modulus of $10.22 \pm 0.16$.

$\mathrm{AO} \mathrm{Vel}$ is located in the region of the Milky Way populated by several $\mathrm{OB}$ associations and sparse open clusters: open cluster $\mathrm{Cr}$ 173, OB association identified by Brandt et al. (1971) and later referred to as Vel OB2, the "Vela sheet" (Eggen 1980, 1983; Loktin et al. 1997; Loktin \& Beshenov 2001) behind which lie several young associations embedded in a dust cloud (Kaltcheva \& Hilditch 2000). AO Vel might be a member of the Vel OB4 association identified by the latter authors (and probably related with the Vel OB2 association of Slawson \& Reed 1988 ), the mean distance of which is $0.72 \mathrm{kpc}$.

We note that if the distance to AO Vel is of the order of $700 \mathrm{pc}$, this system might be resolved astrometrically as a double star. The maximum separation between the two spectroscopic binaries would be then about $27 \mathrm{AU}$, corresponding to about 0.04 arcsec. This is too small for the Hipparcos mission which took place at the time of conjunction (MJD 47700), not of quadrature - but accessible to interferometers like the VLTI. The maximum projected separation occured on MJD 52740 (April 2003), so that further astrometric observations of this multiple are strongly encouraged.

\section{Conclusions}

Using our recent spectroscopic observations we discovered that the triple system AO Vel with an eclipsing BpSi primary is in fact a remarkable quadruple system close to the ZAMS. The analysis of our data as well as all available published data revealed completely different orbital parameters for this system compared to previous works. We found that both spectroscopic binaries move in a wide eccentric orbit $(e=0.29)$ with a period of $41 \mathrm{yr}$ and this orbit is fully consistent with the observed difference between the center-of-mass radial velocities of the two spectroscopic binaries. The calculated orbit imposes a lower limit to the total mass of the binary $\mathrm{C}+\mathrm{D}: M_{\mathrm{C}}+M_{\mathrm{D}} \geq 4.9 M_{\odot}$. The analysis of the stellar parameters and of the evolutionary state of the components reveals their extreme youth. Since no magnetic field measurements have been performed for this system, we have applied for VLT time to obtain spectra in circular polarized light with FORS 1, which is equipped with polarization analyzing optics.

We also plan to obtain additional high resolution, high signal-to-noise spectra of this system at different orbital phases, in order to improve the determination of the stellar parameters and atmospheric elemental abundances of all companions. This unique system is especially important to test evolutionary models of very young stars of intermediate mass. Detailed observations during primary eclipses will provide strong constraints on the distribution of the abundance anomalies on the surface of the A component, complementing the standard Doppler imaging technique based on rotation.

Acknowledgements. We thank Claudio Melo for the reduction of one FEROS spectrum.

\section{References}

Acke, B., \& Waelkens, C. 2004, A\&A, 427, 1009

Bernasconi, P. A. 1996, A\&AS, 120, 57

Bidelman, W. P., \& MacConnell, D. J. 1973, AJ, 78, 687

Brandt, J. C., Stecher, T. P., Crawford, D. L., \& Maran, S. P. 1971, ApJ, 163, L99

Carrier, F., North, P., Udry, S., \& Babel, J. 2004, A\&A, 394, 151

Claret, A., \& Giménez, A. 1992, A\&AS, 96, 255

Clausen, J. V., Giménez, A., \& van Houten, C. J. 1995, A\&AS, 109, 425

Eggen, O. J. 1980, ApJ, 238, 627

Eggen, O. J. 1983, AJ, 88, 197

Gerbaldi, M., Floquet, M., \& Hauck, B. 1985, A\&A, 146, 31

González, J. F., \& Levato, H. 2006, A\&A, 448, 283

Grønbech, B. 1987, A\&AS, 68, 317

Hensberge, H., Nitschelm, C., Freyhammer, L. M., et al. 2004, ASP Conf. Ser., 318, 309

Hubrig, S., North, P., \& Mathys, G. 2000, ApJ, 539, 352

Hubrig, S., North, P., \& Szeifert, T. 2005, Evolution of Magnetic Fields in Stars Across the Upper Main Sequence: Results from Recent Measurements with FORS 1 at the VLT, in Astronomical Polarimetry: current status and future directions, ed. A. Adamson, ASP Conf. Ser., in press

Kaltcheva, N. T., \& Hilditch, R. W. 2000, MNRAS, 312, 753

Kupka, F., Piskunov, N., Ryabchikova, T. A., Stempels, H. C., \& Weiss, W. W. 1999, A\&AS 138, 119

Kurucz, R. L. 1993, CDROM13, SAO Cambridge

Loktin, A. V., \& Beshenov, G. V. 2001, Astron. Lett., 27, 386

Loktin, A., Zakharova, P., Gerasimenko, T., \& Malisheva, L. 1997, Baltic Ast., 6, 316

North, P. 1998, Highlights of Astronomy Vol. 11A, p. 657

North, P., \& Debernardi, Y. 2004, ASPC, 318, 297

Oosterhoff, P. Th., \& van Houten, C. J. 1949, BAN, Vol. 11, 63

Piskunov, N. E. 1992, in Stellar magnetism, ed. Yu. V. Glagolevskij, \& I. I. Romanyuk (St. Petersburg: Nauka), 92

Pojmanski, G. 2002, Acta Astron., 52, 397

Schaller, G., Schaerer, D., Meynet, G., \& Maeder, A. 1992, A\&AS, 96, 269

Slawson, R. W., \& Reed, B. C. 1988, AJ, 96, 988

Wilson, R. E. 1990, ApJ, 356, 613

Wilson, R. E., \& Devinney, E. J. 1971, ApJ, 166, 605

Wolf, M., \& Zejda, M. 2005, A\&A, 437, 545 